RESEARCH REPORT

\title{
Maternal mental health and child nutritional status in four developing countries
}

\author{
Trudy Harpham, Sharon Huttly, Mary J De Silva, Tanya Abramsky
}

J Epidemiol Community Health 2005;59:1060-1064. doi: 10.1136/jech.2005.039180

See end of article for authors' affiliations .....................

Correspondence to: Dr T Harpham, London South Bank University, 103 Borough Road, London SEl OAA, UK;

t.harpham@lsbu.ac.uk

Accepted for publication 29 July 2005

\begin{abstract}
Objective: To test the hypothesis that maternal common mental disorders (CMD) are associated with poorer child nutritional status in four developing countries (Ethiopia, India, Vietnam, and Peru).

Design: Community based cross sectional survey in 20 sites in each of the four countries. Maternal CMD measured by the self reporting questionnaire 20 items (SRQ20). Potential confounding factors include: household poverty, household composition, maternal characteristics such as age and education, child characteristics such as birth weight, age, and sex. Possible mediating factors included the child's physical health and breast feeding status.

Setting: Urban and rural, poor and middle income areas in each country.

Participants: 2000 mothers and their children aged 6-18 months in each country.

Main outcome measures: Child stunting and underweight measured using standard anthropometric techniques.

Results: Levels of maternal CMD and child malnutrition are high in each study setting. After adjusting for confounding factors, the odds ratios (OR) for the association of maternal CMD with child stunting are: India $1.4(95 \% \mathrm{Cl} 1.2$ to 1.6$)$, Peru 1.1 (0.9 to 1.4), Vietnam 1.3 (0.9 to 1.7$)$, and Ethiopia $0.9(0.7$ to 1.2$)$. For child underweight, the confounder adjusted ORs are: India 1.1 (0.9 to 1.4), Peru 0.9 (0.6 to 1.2), Vietnam $1.4(1.1$ to 1.8$)$, and Ethiopia $1.1(0.9$ to 1.4$)$. No clear evidence for effect modification by the child's age or sex was found. Possible mediating factors for the effect of maternal CMD on child malnutrition did not provide strong suggestions for potential mechanisms.

Conclusions: There was a relation between high maternal CMD and poor child nutritional status in India and Vietnam. However, the findings from Peru and Ethiopia do not provide clear evidence for a similar association being present in non-Asian countries. Regardless of the direction of the relation, child nutrition programmes in Asia should consider incorporating promotion of maternal mental health.
\end{abstract}

A though rates of child malnutrition in many poor regions of the world have declined in recent years, ${ }^{1}$ it remains one of the most significant child health problems with an estimated 53\% of child deaths per year attributable to being underweight. ${ }^{2}$ Childcare practices are recognised as a key underlying cause. ${ }^{3}$ The demands of adequate child care are such that it has been suggested that poor mental health of caregivers might adversely affect their child's health and development and recent evidence from South Asia has shown an association between postnatal depression and impaired child growth. ${ }^{4}$ In Goa, malnourished children had a risk 2.3 (95\% CI 1.1 to 4.7 ) times higher than non-malnourished children of having a depressed mother, ${ }^{5}$ in Tamil Nadu the odds were 7.4 ( 1.6 to 38.5 ) times higher, ${ }^{6}$ and in Pakistan odds were 3.9 (1.9 to 7.8 ) times greater. ${ }^{7}$ With reported prevalence rates of maternal depression of $20 \%$ or more in these study areas, ${ }^{5-10}$ the potential public health importance of these findings is great. Rahman et al estimate that reductions in the prevalence of maternal depression could lead to a reduction in child growth retardation of up to $30 \% .{ }^{11}$ It might be, therefore, that intervention programmes to improve maternal mental health should be considered as a strategy to combat child malnutrition. ${ }^{4}$ Yet maternal mental health is largely neglected in child health programmes in developing countries. Even the World Health Organisation's high profile Integrated Management of Childhood Illness strategy does not tackle maternal mental health.

To inform this debate, evidence on whether the association described above is consistent in the Indian sub-continent and whether a similar pattern exists elsewhere in the developing world would be valuable. The objective of this paper is to examine the association between maternal mental health and child nutrition in four developing country populations: Ethiopia, India (Andhra Pradesh state), Peru, and Vietnam. All four countries are part of the Young Lives Project, a longitudinal study of childhood poverty (http:// www.younglives.org.uk).

\section{METHODS}

In each of the four countries 20 sites were purposefully selected by a team of local experts to represent a range of regions and living conditions. In each of the 20 sites, mapping and listing of households that contained a child aged 6-18 months was undertaken. As no official up to date lists or electoral rolls were available this mapping was done by observation by fieldworkers and the identification of eligible children was done by door knocking. Altogether 100 households containing mothers with children aged 618 months were randomly selected from the sample list. ${ }^{12}$ There were no exclusion criteria. Data were collected in 2002. A pilot study was done in each country to adapt the common questionnaire to local idioms and expressions. Standard training was given to all interviewers in all countries. This training used the interviewer training manual available under the "Practical Guidelines" part of the Young Lives web site at http://www.younglives.org.uk and aimed at measuring items in the same way across the four countries. Mothers were interviewed in their local language and anthropometric measurements (weight, height) of the

Abbreviations: $C M D$, common mental disorders; SRQ20, self reporting questionnaire 20 items 


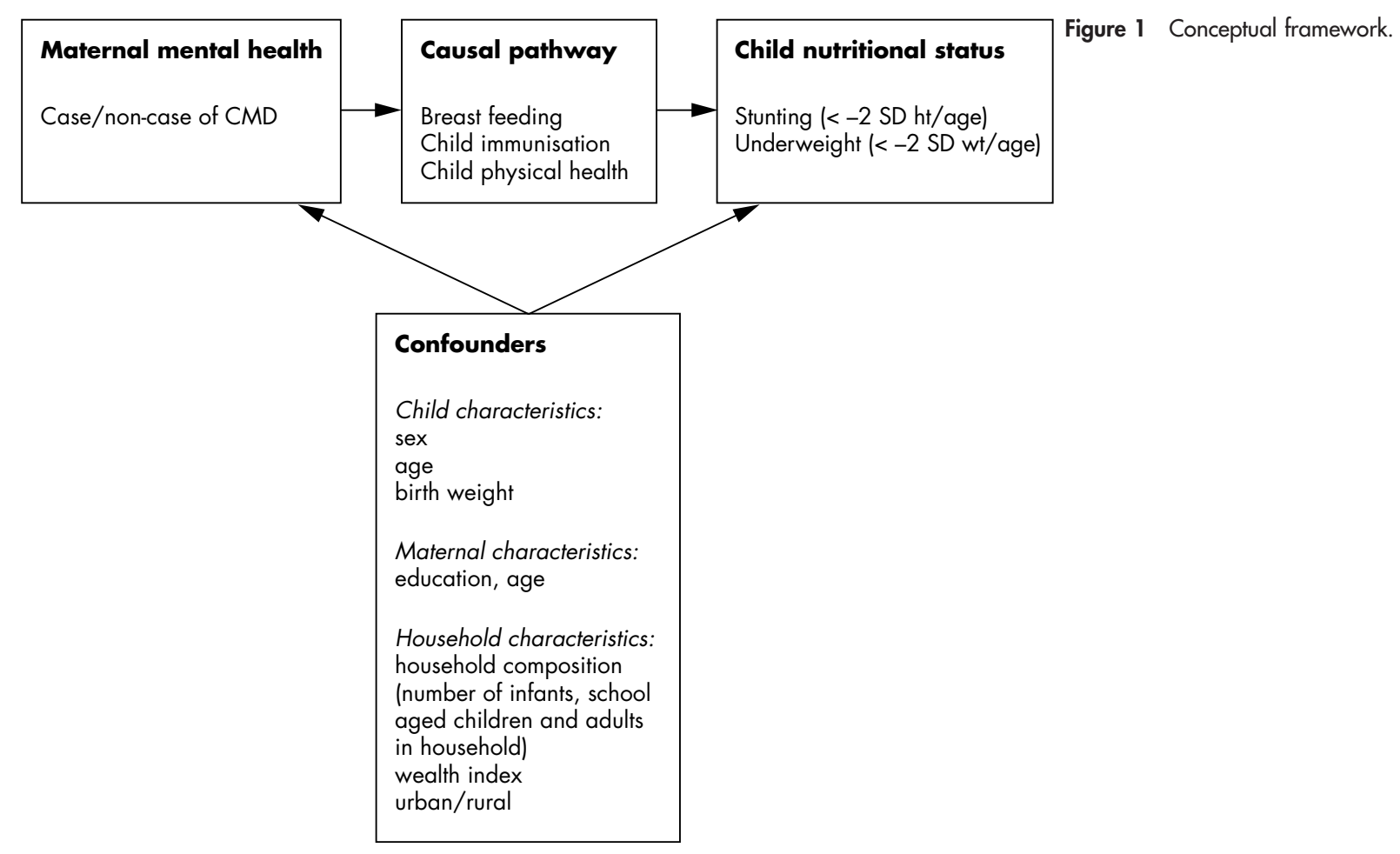

children were taken using the WHO guidelines as presented in the Young Lives interviewer training manual. Data on potential confounding factors (see the conceptual framework in figure 1) were also collected. The response rate was above $90 \%$ in all four countries.

Maternal common mental disorders were considered as the exposure variable, measured by the WHO recommended screening tool the self reporting questionnaire 20 items (SRQ20) that consists of 20 yes/no questions with a reference period of the previous 30 days. It has acceptable levels of reliability and validity in developing countries. ${ }^{13}$ The SRQ20 is not diagnostic and cannot separate out anxiety from depression. Cut off scores to determine how many yesanswers constitute a case have been validated against clinical assessments in each of the study countries. ${ }^{13-15}$ As the validation usually suggested a cut off of $7 / 8$ to separate probable non-cases/cases of CMD that cut off was used in this study.

The anthropometric outcomes were stunting (height for age $\mathrm{Z}$ score $<-2$ ) and underweight (weight for age $\mathrm{Z}$ score $<-2$ ). $\mathrm{Z}$ scores were calculated using EpiInfo EPINUT, and based on the 1977 NCHS reference growth charts ${ }^{16}$ that were adopted for use by the WHO for international comparisons between populations. ${ }^{17}$ Wasting (low weight for height) was not used as an outcome measure because it reflects more acute (short term) conditions and changes such as recent physical morbidity.

A range of potential confounders in the relation between maternal CMD and child malnutrition were considered. Information was collected through a household questionnaire that was administered to the caregiver/mother of the child. Data on child characteristics and health were crosschecked against documentation where possible.

Household factors included various variables relating to household composition, a measure of wealth, and urban/ rural location.

Maternal characteristics comprised maternal age (in five-year bands) and education. The latter was classified as whether or not the mother had completed primary education.
Child characteristics considered were sex, age (under or over 12 months), and birth weight (under or over $2500 \mathrm{~g}$ ).

Factors that we hypothesised may mediate the effect of CMD on child malnutrition were also considered. These were child vaccination status (depressed mothers may not get their children vaccinated), child physical health (depressed mothers may not seek care for sick children), and breast feeding status (depressed mothers may stop breast feeding). With respect to the latter, the mother was asked if she ever breast fed and if so the duration of breast feeding. Responses to these two questions were then combined to create a variable with three categories: still breast feeding; stopped breast feeding; and never breast fed. Reported duration for those who had stopped was considered to be of limited use in this analysis as (in addition to the possibility of recall bias) the vast majority of children were still breast feeding.

Separate logistic regression models (using generalised estimating equations to take into account the clustered nature of the sample) were fitted to estimate the effects of CMD on odds of having a child who was stunted or underweight. Each block of variables from the conceptual framework was added to the models in consecutive stages. We used the same set of variables (decided upon a priori) in each country model to provide some point of comparison between the results. The aim was not to fit the most parsimonious model, but to explore how the addition of other variables affected the relation between maternal CMD and child growth. Finally, we hypothesised that the effect of maternal CMD on child malnutrition might differ according to a child's age or sex, thus interaction coefficients were added to explore this.

\section{RESULTS}

Table 1 presents the prevalence of probable cases of maternal CMD, child stunting, and underweight, and the distributions of the other variables included in the conceptual framework, for the four study populations. 
Table 1 Description of study populations

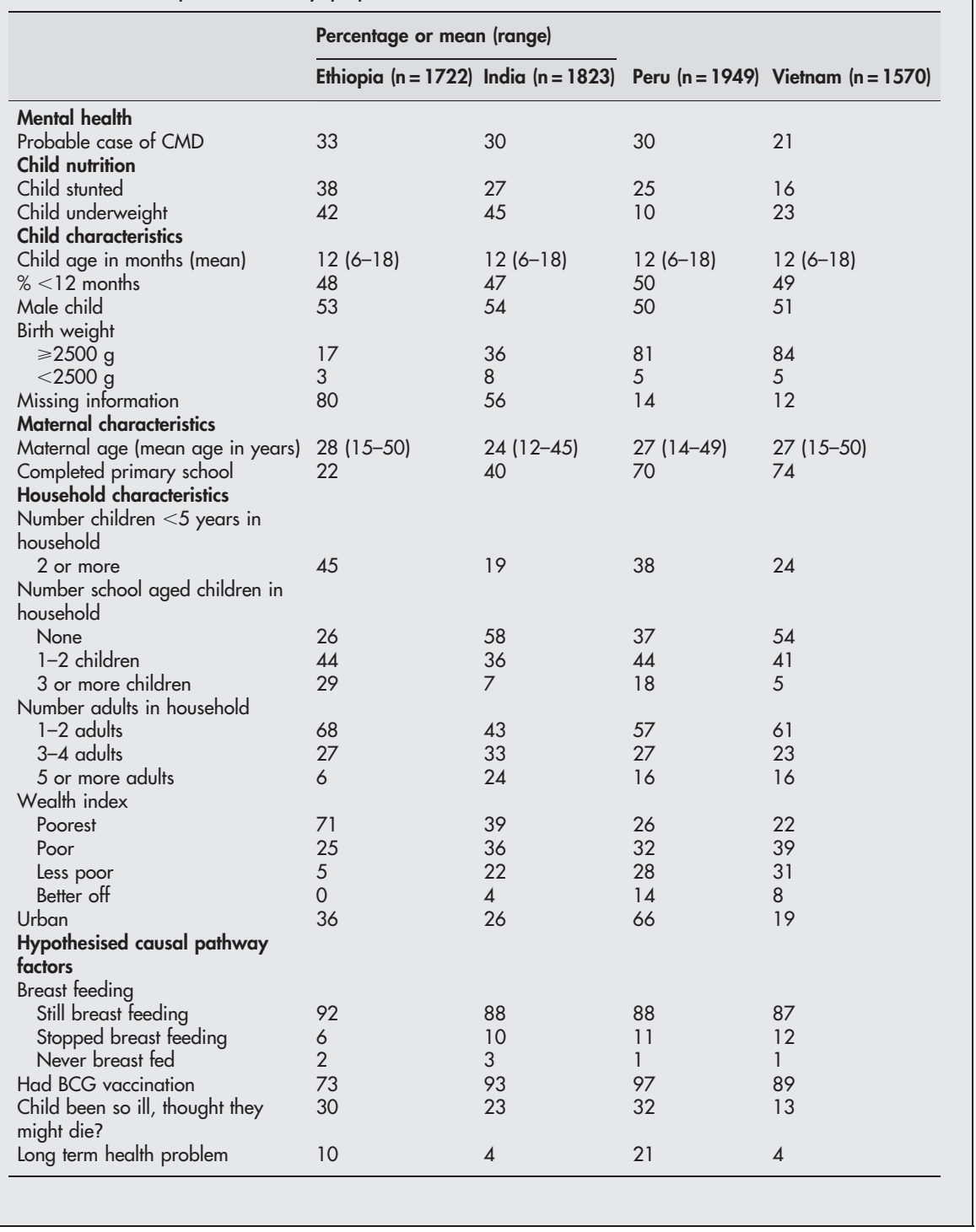

The odds ratios (OR) of the association between maternal CMD and child stunting are presented in table 2, and those for underweight in table 3.

The prevalence of maternal CMD seen in these study populations is fairly typical for women in developing countries, ${ }^{14}$ ranging from $21 \%$ in Vietnam to $33 \%$ in Ethiopia. Levels of malnutrition are high, stunting being most prevalent in Ethiopia (38\%) and least prevalent in Vietnam $(16 \%)$, and underweight status being highest in India $(44 \%)$ and lowest in Peru $(10 \%)$.
In three of the study populations-India, Peru, and Vietnam-mothers with CMD have significantly higher odds of having a stunted child than those without CMD (crude ORs of 1.2-1.6). However, there is evidence of confounding by other factors. Adjustment for household factors results in a reduction in the ORs. Further adjustments for maternal and child characteristics have little impact on the ORs. Adjusted for all confounders (model 4), the positive association persists in India, Peru, and Vietnam, but is only statistically significant in India where mothers suffering from CMD have

Table 2 Association between maternal CMD and stunting in children. Crude OR (model 1), OR adjusted for household variables (model 2), additionally adjusted for maternal characteristics (model 3), additionally adjusted for child characteristics (model 4), additionally including variables on causal pathway (model 5)

\begin{tabular}{lllll}
\hline & OR $(95 \%$ confidence interval) & & \\
\cline { 2 - 5 } & Model 1 (crude) & Model 2 & Model 3 & $\begin{array}{l}\text { Model } 4 \text { (adjusted for all } \\
\text { confounders) }\end{array}$ \\
\hline Ethiopia $(n=1723)$ & $0.9(0.8$ to 1.2$)$ & $0.9(0.7$ to 1.2$)$ & $0.9(0.7$ to 1.2$)$ & $0.9(0.7$ to 1.2$)$ \\
India $(n=1823)$ & $1.6(1.3$ to 1.9$)$ & $1.5(1.2$ to 1.7$)$ & $1.5(1.3$ to 1.7$)$ & $1.4(1.2$ to 1.6$)$ \\
Peru $(n=1949)$ & $1.2(1.0$ to 1.5$)$ & $1.2(1.0$ to 1.4$)$ & $1.1(0.9$ to 1.4$)$ & $1.1(0.9$ to 1.4$)$ \\
Vietnam $(n=1570)$ & $1.4(1.1$ to 1.7$)$ & $1.2(1.0$ to 1.6$)$ & $1.3(1.0$ to 1.6$)$ & $1.3(0.9$ to 1.7$)$ \\
\hline
\end{tabular}


Table 3 Association between maternal CMD and underweight in children. Crude OR (model 1), OR adjusted for household variables (model 2), additionally adjusted for maternal characteristics (model 3), additionally adjusted for child characteristics (model 4), additionally including variables on causal pathway (model 5)

\begin{tabular}{|c|c|c|c|c|c|}
\hline & \multicolumn{5}{|c|}{ OR ( $95 \%$ confidence interval) } \\
\hline & Model 1 (crude) & Model 2 & Model 3 & $\begin{array}{l}\text { Model } 4 \text { (adjusted for all } \\
\text { confounders) }\end{array}$ & Model 5 \\
\hline Ethiopia $(n=1723$ ) & $1.2(1.0$ to 1.4$)$ & 1.1 (0.9 to 1.4$)$ & 1.1 (0.9 to 1.4$)$ & 1.1 (0.9 to 1.4$)$ & $1.1(0.9$ to 1.3$)$ \\
\hline India $(n=1823)$ & $1.3(1.1$ to 1.7$)$ & $1.2(1.0$ to 1.5$)$ & $1.2(0.9$ to 1.5$)$ & $1.1(0.9$ to 1.4$)$ & $1.1(0.9$ to 1.3$)$ \\
\hline Peru $(n=1949)$ & $1.1(0.8$ to 1.4$)$ & 0.9 (0.7 to 1.2$)$ & $0.9(0.7$ to 1.2$)$ & $0.9(0.6$ to 1.2$)$ & $0.8(0.6$ to 1.1$)$ \\
\hline Vietnam $(n=1570)$ & $1.5(1.2$ to 1.9$)$ & $1.4(1.1$ to 1.8$)$ & $1.4(1.1$ to 1.8$)$ & $1.4(1.1$ to 1.8$)$ & $1.3(1.0$ to 1.7$)$ \\
\hline
\end{tabular}

odds of having a stunted child 1.4 (95\%CI: 1.2 to 1.6$)$ times higher than that of mothers without CMD. This pattern was not seen in the Ethiopian sample.

Inclusion of the potential mediating variables in the model (model 5) does not change the ORs, suggesting that in these study populations they are not important pathways through which CMD has an effect on stunting.

In all four study populations, mothers with CMD have higher odds of having an underweight child than those without CMD (crude ORs of 1.0-1.5). Adjustment for household factors attenuates the association, with little further attenuation after adjustment for maternal and child characteristics. After adjustment for all potential confounders (model 4), there is little evidence of an association between CMD and underweight except in Vietnam $(1.4 ; 95 \%$ CI 1.1 to $1.8)$.

Inclusion of the potential mediating variables in the model (model 5) only slightly attenuates the effect of CMD on underweight status - in Vietnam the OR changes to 1.3 (1.0 to 1.7)—suggesting possible mechanisms for how maternal CMD asserts an effect on child underweight status.

No statistically significant interactions were found between infant sex or age and maternal CMD for either nutritional outcome. However in Peru, Vietnam, and India, the OR for the effect of CMD on stunting was always closer to 1 among female children than males and among those children aged less than 12 months than older children. No such consistent patterns of effect modification by age or sex were found with respect to CMD and underweight status.

\section{DISCUSSION}

This study provides evidence of an association between poor maternal mental health and poor child nutritional status in study populations from India and Vietnam but not from Peru

\section{What this paper adds}

What is already known on this subject

Women in developing countries have high rates of common mental disorders (CMD). Recent evidence from South Asia suggests that poor maternal mental health is associated with poor child growth. This finding has potentially important policy implications for improving child nutrition in developing countries but we were unsure if the findings would be consistent in that region and also whether the same pattern would be found in other developing countries.

\section{What this study adds}

Even when controlling for a particularly wide range of factors we found that mothers with poor mental health in India and Vietnam have significantly increased odds of having malnourished children. However, the association was less clear in Peru and Ethiopia.

\section{Policy implications}

Mental health is gradually getting onto the public health agenda in developing countries. The evidence from this study confirms that promotion of maternal mental health may be important for the improvement of child nutrition. Although the causal direction of the relation between maternal mental health and child growth is not yet established, child nutrition programmes in Asia need to consider incorporating promotion of maternal mental health. This needs to include detection and treatment of CMDs at the primary care level and the addition of preventive actions (strengthening social support for women) within community development programmes. Future research should consider whether poor maternal mental health is also associated with other child health outcomes such as physical or mental morbidity.

and Ethiopia. It thus confirms previous evidence from the Asian region..$^{5-7}$ Despite similar levels of CMD and substantial levels of child malnutrition, it is interesting to find no such association in the Peruvian and Ethiopian study populations. This heterogeneity in the results is particularly interesting and needs to be further analysed to fully understand the association between maternal mental health and child nutrition. As this is a new result there is no existing literature that explains this differential pattern. Qualitative research is now needed to investigate the reasons for this difference that may, for example, be related to the special and particularly pressurised cultural role of women in relation to childcare in Asia. Recent literature discusses the multiple roles of Asian women (productive and reproductive, household, and community), the expectations of family members (especially mothers in law), and suggests that mothering is a "site for disempowerment" in Asia. ${ }^{18}$ Such disempowerment may be exacerbated by a "failing" child and might directly affect mental health. Does such disempowerment not happen outside Asia? This will be a fruitful area for future qualitative research.

\section{Strengths and weaknesses of the study}

This is the first time that data from four countries from different regions of the world, using the same data collection methods, have been compared to test the consistency of the recently observed relation between maternal mental health and child growth. Previous studies had been limited to the Indian sub-continent but this study was able to examine two countries in Asia plus Peru and Ethiopia. The study was able to adjust for a particularly wide range of potential confounding factors that the other studies were unable to do. Generalised estimating equations adjust for the clustered nature of data and provide a more stringent test of the association. 
The previously cited studies in Asia ${ }^{8-10}$ have examined the association between postnatal depression and malnutrition in infants. This paper looks at mental health among mothers of a broader age range of children and therefore will include both cases of postnatal depression and other types of CMD. In exploring interactions between CMD and age we found that the association between this more wide ranging exposure and malnutrition is also evident among older children.

As with other studies on this topic, the cross sectional nature of this analysis precludes causal understanding. Additionally, the reference periods of the outcome and exposure measures may further complicate causal inference- the child nutrition indicators are long term and while the SRQ20 is likely to capture women who have had CMD for a longer period, its reference period is one month. Children's failure to thrive may cause mental distress to their mothers and has been associated with postnatal depression in Australia ${ }^{19}$ and Turkey. ${ }^{20}$ Equally, maternal mental ill health may impede care of children thus leading to poor growth. In a longitudinal study to examine this association, Rahman et al ${ }^{11}$ found that children of prenatally depressed mothers showed significantly more growth retardation than controls at all time points. Young Lives is also a longitudinal study and the same mothers and their children will be followed up for 15 years thus enabling a better understanding of the causal nature of this relation.

\section{Implications and future research}

Mental health is gradually getting onto the public health agenda in developing countries. The evidence from this study confirms that promotion of maternal mental health may be important for the improvement of child nutrition. The direction of the relation may be that sickly children cause depression and anxiety in mothers or it may be that depressed and anxious mothers are unable to function effectively to maintain good nutrition of their children. This latter link might be related to impaired or decreased breast feeding and/or lack of social support to adequately care for a child. Regardless of the direction of the association, existing community based nutrition programmes, which are abundant in developing countries, can usefully add maternal mental health to their agenda. So, although the causal direction of the relation between maternal mental health and child growth is not yet established, child nutrition programmes in Asia need to consider incorporating promotion of maternal mental health. This needs to include detection and treatment of CMDs at the primary care level and the addition of preventive actions (strengthening social support for women) within community development programmes. Future research should consider whether poor maternal mental health is also associated with other child health outcomes such as physical or mental morbidity.

\section{ACKNOWLEDGEMENTS}

The authors are grateful to the numerous members of the Young Lives team who contributed to the design and conduct of the project, to the cohort participants, and their families in each country.

\section{Authors' affiliations}

T Harpham, London South Bank University, London, UK

S Huttly, M J De Silva, T Abramsky, London School of Hygiene and

Tropical Medicine, London, UK

Funding: the UK Department for International Development (DFID) funded the first phase of the project. Mary De Silva is funded by a MRC Studentship.

Conflicts of interest: the researchers are independent from the funder. There are no competing interests to declare.

Ethics approval: granted by ethics committees of The London School of Hygiene and Tropical Medicine, London South Bank University, Reading University, and local ethics committees in each of the four developing countries.

\section{REFERENCES}

1 de Onis $M$, Blossner $M$, Borghi $E$, et al. Methodology for estimating regional and global trends of child malnutrition. Int J Epidemiol 2004;33:1260-70.

2 Black RE, Morris SS, Bryce J. Where and why are 10 million children dying every year? Lancet 2003;361:2226-34.

3 Unicef. The state of the world's children. New York: UNCs, Fund, 1998.

4 Patel V, Rahman A, Jacob KS, et al. Effects of maternal mental health of infant growth in low-income countries: new evidence from South Asia. BMJ 2004;328:820-3.

5 Patel V, Rodrigues M, De Souza N. Gender, poverty and post-natal depression: a cohort study from Goa, India. Am J Psychiatry 2002;159:43-7

6 Chandran M, Tharyan P, Muliyil J, et al. Post-partum depression in a cohort of women from a rural area of Tamil Nadu, India. Incidence and risk factors. Br J Psychiatry 2002;181:499-504.

7 Rahman A, lqbal Z, Harrington R. Life events, social support and depression in childbirth: perspectives from a rural community in the developing world. Psychol Med 2003;33:1161-7.

8 Rahman A, Love H, Bunn J, et al. Mothers' mental health and infant growth: a case control study from Rawalpindi, Pakistan. Child Care Health Dev 2004;30:21

9 Patel V, De Souza N, Rodrigues M. Postnatal depression and infant growth and development in low-income countries: a cohort study from Goa, India. Arch Dis Child 2003;88:34-7.

10 Anoop S, Saravan B, Joseph A, et al. Maternal depression and low maternal intelligence as risk factors for malnutrition in children: a community case-control study from South India. Arch Dis Child 2004;89:325-9.

11 Rahman A, Bunn IZ, Lovel H, et al. Impact of maternal depression on infant nutritional status and illnesses: a cohort study. Arch Gen Psychiatry 2004;61:946-52.

12 Wilson IM, Huttly SRA, Fenn B. A case study of sample design for longitudinal research: Young Lives. International Journal of Social Research Methodology (in press).

13 WHO. A user's guide to self-reporting questionnaires. Geneva: Division of mental health, WHO, 1994

14 Harpham T, Reichenheim M, Oser R, et al. How to do (or not to do)... Measuring mental health in a cost-effective manner. Health and Policy Planning 2003; 18:344-9.

15 Tuan T, Harpham T, Huong N. Validity and reliability of the self- reporting questionnaire 20 items (SRQ20) in Vietnam. Hong Kong Journal of Psychiatry 2004; 14:15-18.

16 Hamill PVV, Drizid TA, Johnson CL, et al. NCHS growth curves for children birth-18 years. Hyattsville, MD: USHEW-PHS, 1977.

17 Waterlow JC, Buzina R, Keller W, et al. The presentation and use of height and weight data for comparing the nutritional status of groups of children under the age of 10 years. Bull W H O 1977;55:489-98.

18 Asia Research Institute. International workshop on working and mothering: Asian women negotiating work challenges and family commitments, 29-31 Jan 2004, Asia, Research Institute, National University of Singapore, 2004.

19 Davis L, Edwards $\mathrm{H}$, et al. The impact of very premature birth on the psychological health of mothers. Early Human Development 2003;73:61-70. 20 Inandi TO, Elci C, et al. Risk factors for depression in postnatal first year, in eastern Turkey. Int J Epidemiol 2002;31:1201-7. 